\title{
Photon Strength Functions of Heavy Nuclei: Achievents and Open Problems
}

\author{
F. Bečváŕr \\ Charles University in Prague, Faculty of Mathematics and Physics \\ V Holešovičkách 2, 18000 Prague 8, Czech Republic \\ E-mail: becvar@mbox.troja.mff.cuni.cz
}

Systematic experimental studies of decay of highly excited levels in heavy nuclei started as early as 50 years ago, mostly by analyzing the spectra of $\gamma$ rays following the capture of slow neutrons. Soon, it turned out that the $\gamma$ decay of these nuclei is strongly dominated by the $E 1$ transitions. Further studies revealed that the $E 1$ decay rate is closely linked via the principle of the detailed balance to the giant dipole resonances built - in line with Brink hypothesis and the paradigm of photon strength - not only on the ground state, but also on each excited level. These findings brought the first evidence for the electric giant dipole vibrations coupled to the excited levels and demonstrated viability of Brink's concept. Nevertheless, with growing information on $\gamma$ rays following neutron capture it gradually emerged that extrapolation of the photonuclear cross sections to the energy region below the neutron threshold leads to overpredictions of the $E 1$ photon strength function (PSF), especially in case of spherical and transitional nuclei. Several theories were developed, but this disproportion has not yet been satisfactorily accounted for. New information deduced from the state-of-the-art NRF experiments seems promising for further studies of the $E 1$ PSFs. Regarding $M 1$ transitions, in accordance with the single-particle model, until the end of 80's it had been assumed that the M1 PSF was energy independent. However the later data from $\left(\mathrm{p}, \mathrm{p}^{\prime}\right),\left({ }^{3} \mathrm{He}^{3} \mathrm{He}^{\prime} \gamma\right)$ and $\left({ }^{3} \mathrm{He}, \alpha \gamma\right)$ reactions together with data on two-step cascades following the thermal neutron capture clearly indicated resonance-like structures in the M1 PSFs for a number of nuclei. It is believed that these structures belong to the scissors $M 1$ vibrational mode, which implies that, like in the case of the $E 1$ radiation, this mode is coupled to any excited nuclear level. But still, many questions remain unanswered. A brief overview of the present knowledge on PSFs will is given and the main problems to be solved are formulated.

Workshop on Photon Strength Functions and Related Topics

June 17-20 2007

Prague, Czech Republic

\footnotetext{
*Speaker.
} 